\title{
Effect of Saturating the Electrolyte with Oxygen on the Activity for the Oxygen Evolution Reaction
}

\author{
Amanda C. Garcia and Marc T. M. Koper*(e) \\ Leiden Institute of Chemistry, Leiden University, PO Box 9502, 2300 RA, Leiden, The Netherlands
}

Supporting Information

\section{INTRODUCTION}

With the increased interest in the electrochemical conversion of renewable electricity, water, and carbon dioxide to fuels, there is an ever-growing number of papers reporting new electrocatalysts for the oxygen evolution reaction (OER). ${ }^{1-5}$ The OER is the anode reaction in the electrolysis of water and $\mathrm{CO}_{2}$ and a major source of efficiency loss, because of its high overpotential. ${ }^{6}$ To meaningfully compare the activity of the many new materials that are currently synthesized and tested, it is important that the research community agrees on proper standardization, benchmarking, and best practices 7,8

Several papers reporting on the activity of OER state that it is necessary to saturate the electrolyte with oxygen gas before measurement, in order for the electrode "to reach its rest potential" or "to fix the equilibrium potential", 2,9-14 and saturating the solution with oxygen seems to have become an often-employed practice (see, e.g., refs 15-18). A recent paper claimed that oxygen in the electrolyte may reduce the OER activity on nickel (supported on graphene) and change the Tafel slope via a van der Waals-type interaction of molecular oxygen with the active site, hampering access by hydroxide ions. ${ }^{11}$ The argument to fix the equilibrium potential is based on the idea that, in the absence of oxygen, the driving force for oxygen evolution should be higher than in its presence. However, at a given electrode potential, the rate of oxygen evolution itself must be independent of whether $\mathrm{O}_{2}$ is present in solution or not. What potentially changes in the presence of oxygen is the rate of the back reaction, i.e., the oxygen reduction reaction (ORR), and therefore the net production rate of oxygen may be dependent on whether $\mathrm{O}_{2}$ is present or not. This could play a role for a reversible reaction near its equilibrium potential, but it should be irrelevant for an irreversible reaction such as OER (ORR rates can safely be neglected above $1.3 \mathrm{~V}$ ). The rate of an electrode reaction at a given applied potential is more accurately measured in the absence of the product, regardless of whether, under the experimental conditions, the equilibrium potential is welldefined or not. This also implies that the overpotential, when defined as the difference between the applied potential and the equilibrium potential, is not well-defined in the absence of oxygen in solution, but this has no (theoretical) effect on the rate of the OER at a given applied potential. Of course, close to equilibrium, it may still be necessary to correct for any current due to the back reaction if one wants to know the rate of the forward reaction only. The notion that the presence of $\mathrm{O}_{2}$ in solution may have an effect on the state of the surface (and thereby influence its chemistry and "rest potential") has been studied for platinum electrodes. ${ }^{19,20}$ It was found that $\mathrm{O}_{2}$ in solution may indeed have an effect on the activity and stability of platinum electrodes, but apparently only under potential cycling conditions, or at relatively negative potentials, suggesting that oxygen reduction may play a role. Kongkanand and Ziegelbauer concluded that the effect of $\mathrm{O}_{2}$ in solution on the oxide coverage on platinum is negligible. ${ }^{19}$

To clarify the experimental role of oxygen saturation of the electrolyte on OER activity, we decided to study the effect of electrolyte oxygen on the OER on Pt and Ni-oxyhydroxide electrodes. In this Viewpoint, we show that (i) oxygen dissolved in the electrolyte has no significant effect on the OER activity (at least not on Pt-oxide and Ni-oxyhydroxide surfaces) and (ii) in the standardization of best practices for OER studies, care should be taken in employing a proper (placement of the) reference electrode, and in taking measures that small oxygen bubbles are efficiently removed from the electrocatalyst surface (for instance, by rotation).

\section{EXPERIMENTAL RESULTS}

Although platinum is not the best catalyst for the OER, we use it in this work as a model system to provide insight into the effect of different parameters. Figure 1 shows the current-

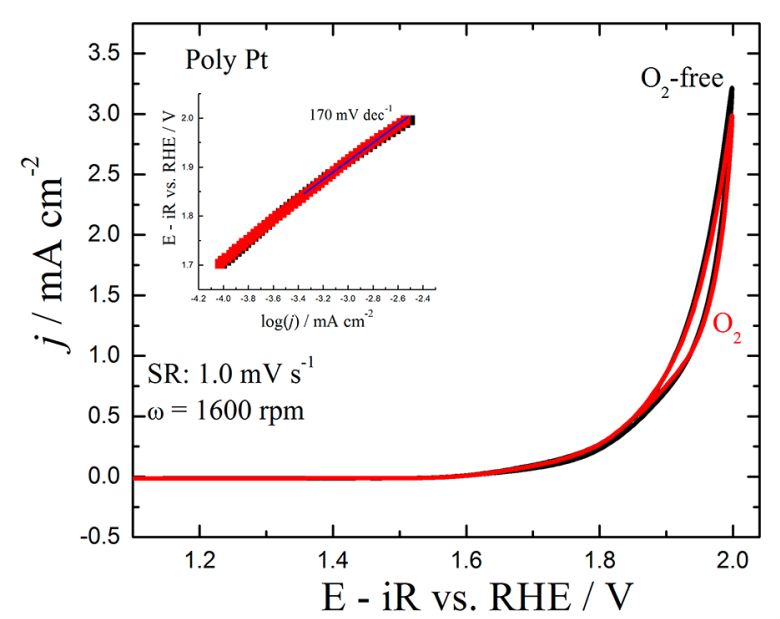

Figure 1. Cyclic voltammetry for polycrystalline $\mathrm{Pt}$ in $\mathrm{pH} 13$ in the region of oxygen evolution reaction under rotation of the electrode at $1600 \mathrm{rpm}$. Scan rate $=1.0 \mathrm{mV} \mathrm{s}^{-1}$. Measurements were performed using an $\mathrm{Ag} / \mathrm{AgCl}$ reference electrode. [Legend: red line denotes electrolyte saturated with $\mathrm{O}_{2}$; black line denotes electrolyte saturated with $\mathrm{Ar}$, with no $\mathrm{O}_{2}$ present.]

Received: April 12, 2018

Published: August 21, 2018 
potential profiles obtained during water oxidation catalyzed by polycrystalline $\mathrm{Pt}$ at $\mathrm{pH} \mathrm{13}$, with the electrolyte saturated with $\mathrm{O}_{2}$ (red line) and the electrolyte obtained by removing oxygen from the solution (black line), under rotation of the electrode at $1600 \mathrm{rpm}$ and at a scan rate of $1.0 \mathrm{mV} \mathrm{s}^{-1}$. The onset potential of the OER is $\sim 1.57 \mathrm{~V}$ vs RHE and no significant difference are observed between the $\mathrm{O}_{2}$-saturated solutions and the $\mathrm{O}_{2}$-free solutions. Under both conditions, the $i R$-corrected Tafel plots (see inset in Figure 1) show a similar slope of 170 $\mathrm{mV} \operatorname{dec}^{-1}$, in agreement with the literature. ${ }^{21}$ The same insensitivity to $\mathrm{O}_{2}$ saturation was observed in acidic solution (result not shown).

Figure 2 compares the effect of the rotation for the same curves showed in Figure 1. There is a clear effect of the

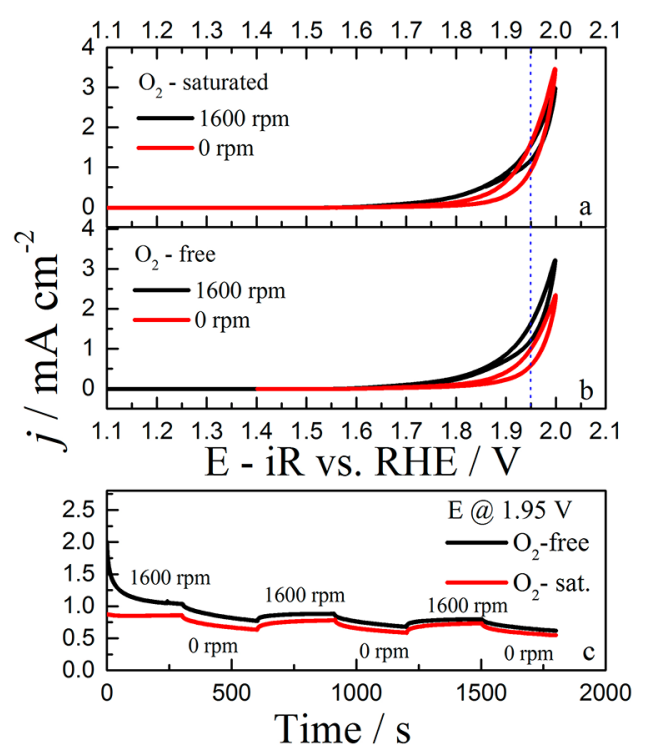

Figure 2. Cyclic voltammetry for polycrystalline $\mathrm{Pt}$ in $\mathrm{pH} 13$ in the region of oxygen evolution reaction. (a) $\mathrm{O}_{2}$-saturated and (b) $\mathrm{O}_{2}$-free solution at $1600 \mathrm{rpm}$ and without rotation. Scan rate $=1.0 \mathrm{mV} \mathrm{s}^{-1}$, using a $\mathrm{Ag} / \mathrm{AgCl}$ reference electrode. (c) Chronoamperometry curves at $E=1.95 \mathrm{~V}$ vs RHE. [Legend: red line represents the presence of $\mathrm{O}_{2}$; black line represents the absence of $\mathrm{O}_{2}$.]

rotation rate of the $\mathrm{RDE}$, comparing OER current-voltage curves and chronoamperometry measurements with and without rotation $(\omega=1600$ and $0 \mathrm{rpm}$, respectively). Figures $2 \mathrm{a}$ and $2 \mathrm{~b}$ show that, in the absence of rotation, the onset of OER is delayed, both for the $\mathrm{O}_{2}$-saturated and $\mathrm{O}_{2}$-free solutions. We ascribe this observation to the more-efficient removal of oxygen bubbles from the electrode in the presence of rotation. However, at higher potentials, the comparison is more difficult to rationalize, because, in the $\mathrm{O}_{2}$-saturated solution, the absence of rotation leads to a higher current. We note that voltammetric OER rates are generally not perfectly reproducible when the electrode is not rotated. To add to the complication of comparing these curves, the effects are dependent on the scan rate. At $10 \mathrm{mV} \mathrm{s}^{-1}$, we observe no difference between rotation and no rotation for both electrolytes, whereas, at $50 \mathrm{mV} \mathrm{s}^{-1}$, the OER current is higher in a stagnant $\mathrm{O}_{2}$-free solution (these data are shown in Figure $\mathrm{S} 1$ in the Supporting Information). To eliminate this effect of scan rate, we also performed chronoamperometry experiments at a fixed potential of $1.95 \mathrm{~V}$ (vs RHE). Figure $2 \mathrm{c}$ compares these experiments for $\mathrm{O}_{2}$-saturated (red curve) and $\mathrm{O}_{2}$-free solutions (black curve). In the beginning of the experiment, when both electrodes are rotated, the $\mathrm{O}_{2}$-free solution gives a higher OER current than the $\mathrm{O}_{2}$-saturated solution. We attribute this observation to the more-facile nucleation of oxygen bubbles on the platinum electrode in an $\mathrm{O}_{2}$-saturated solution, thereby blocking the catalytic surface. If rotation is stopped, the OER activity decreases for both electrolytes, and once rotation is started again, the activity increases again. This clearly shows that there is an important effect of rotation, which we tentatively attribute to the enhanced removal of bubbles in the presence of rotation. It is also observed that, at longer times, the OER activities in $\mathrm{O}_{2}$-saturated and (initially) $\mathrm{O}_{2}$-free solutions approach each other.

We note that, in comparing the OER activities in $\mathrm{O}_{2}$ saturated and $\mathrm{O}_{2}$-free electrolytes, it is important to use an oxygen-insensitive reference electrode and to keep oxygen rigorously away from the reference electrode. When performing the experiment using an internal hydrogen reference electrode, we noted that, in an $\mathrm{O}_{2}$-saturated solution, the onset potential for OER is substantially lower than that observed in the absence of oxygen. The reason for this turned out to be a shift in the potential of the hydrogen reference electrode, whose potential now becomes a mixed potential that is determined by hydrogen oxidation and oxygen reduction. The effect is especially significant in alkaline media, where the kinetics of the hydrogen oxidation and hydrogen evolution on platinum are comparatively slow and, hence, more sensitive to spurious side reactions. In any case, we recommend using a $\mathrm{Hg} / \mathrm{HgO}$ or $\mathrm{Ag} / \mathrm{AgCl}$ reference electrode for OER experiments in alkaline media, although caution should be taken with the latter as $\mathrm{Cl}^{-}$ions during long-term measurements can leach out from the reference electrode compartment and diffuse to the working electrode, possibly influencing the result of the electrochemical reaction. Another issue related to the use of the $\mathrm{Ag} / \mathrm{AgCl}$ reference electrode in high $\mathrm{pH}$ is that $\mathrm{Ag}_{2} \mathrm{O}$ or $\mathrm{AgOH}$ will be formed and the electrode potential will be that of a mixed $\mathrm{Ag} / \mathrm{AgCl} / \mathrm{Ag}_{2} \mathrm{O}$ electrode. In addition, the presence of oxygen in the electrolyte is expected to enhance the dissolution of a platinum counter electrode. ${ }^{19,20}$ While this is less of an issue for OER studies on the electrode materials used in this paper, it is of concern when studying electrocatalytic reactions on working electrodes sensitive to small amounts of platinum. ${ }^{8}$

The effect of dissolved oxygen electrolyte toward OER was also investigated using an $\mathrm{NiOOH}$-based electrocatalyst, chosen for its high activity and stability in alkaline media. ${ }^{4,22-24}$ We performed our measurements in Fe-free electrolyte, since Boettcher et al. ${ }^{13}$ have demonstrated that even unintentional Fe impurities (at the parts per billion (ppb) level) in the stock electrolyte are sufficient to enhance the performance of $\mathrm{NiOOH}$.

Figures $3 \mathrm{a}$ and $3 \mathrm{~b}$ show the 10th scan obtained at a rate of $10.0 \mathrm{mV} \mathrm{s}^{-1}$ in the presence and absence of oxygen in the solution under rotation $(1600 \mathrm{rpm})$ and under stationary conditions, respectively, for freshly prepared $\mathrm{NiOOH}$ electrocatalysts. Both voltammograms show the $\mathrm{Ni}(\mathrm{OH})_{2} / \mathrm{NiOOH}$ $\left(\mathrm{Ni}^{\mathrm{II}} / \mathrm{Ni}^{\mathrm{III}}\right)$ redox transition in the potential region of $1.3-1.4$ $\mathrm{V}$ vs RHE. The potential at which this redox transition occurs is not dependent on the presence of $\mathrm{O}_{2}$ in solution $(\mathrm{a} \mathrm{Hg} /$ $\mathrm{HgO}$ electrode was used as a reference electrode). However, we note that, when using an internal RHE as a reference electrode, the redox peak shifted by ca. $0.1 \mathrm{~V}$ to more-negative potential in the presence of oxygen in the solution (see Figure 

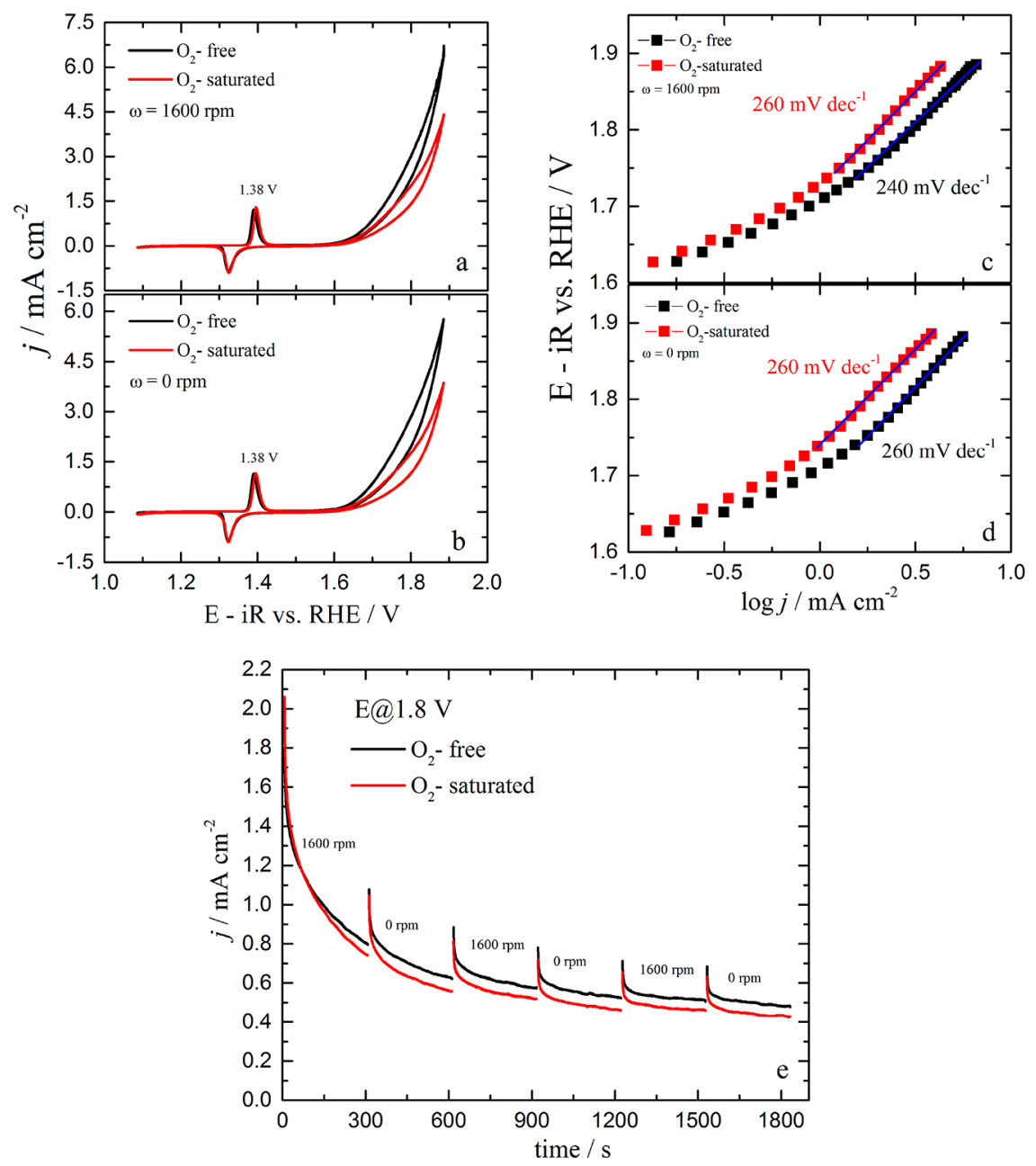

Figure 3. Cyclic voltammetry for $\mathrm{NiOOH}$ at $\mathrm{pH} 13$ in the region of oxygen evolution reaction (a) under rotation of the electrode at $1600 \mathrm{rpm}$ and (b) without rotation, respectively, and in the presence and presence of $\mathrm{O}_{2}$ in solution. (c) and (d) Corresponding Tafel plots for the cyclic voltammograms shown in panels (a) and (b), respectively. (e) Chronoamperometry curves at $E=1.8 \mathrm{~V}$. Scan rate $=10.0 \mathrm{mV} \mathrm{s}^{-1}$. Measurements were performed using $\mathrm{Hg} / \mathrm{HgO}$ reference electrode. [Legend: red line represents the presence of $\mathrm{O}_{2}$; black line represents the absence of $\mathrm{O}_{2}$.]

S2 in the Supporting Information), because of the abovementioned problem of the shift of the reference electrode potential.

An effect of the presence of oxygen was observed in the potential region where the oxygen evolution occurs $(E>1.6 \mathrm{~V}$ vs RHE). Figures $3 a$ and $3 b$ compare the OER curves in the absence and presence of oxygen at 1600 and $0 \mathrm{rpm}$, respectively. The results show that the OER activity becomes slightly lower by saturating the solution with $\mathrm{O}_{2}$. The Tafel slopes of the anodic scan for $\mathrm{NiOOH}$ at $1600 \mathrm{rpm}$ (Figure 3c), in the presence and absence of oxygen, show values of 260 and $240 \mathrm{mV} \mathrm{dec}{ }^{-1}$, respectively. For $\mathrm{NiOOH}$, in the absence of electrode rotation (Figure $3 \mathrm{~d}$ ), the currents are generally lower than in the presence of rotation (compare Figures $3 a$ and $3 b$ ) but the Tafel slopes are similar. The results show that the highest OER activity is obtained in an $\mathrm{O}_{2}$-free electrolyte under rotation of the electrode. The difference with the activity in an $\mathrm{O}_{2}$-saturated electrode in the absence of rotation can be almost a factor of 2 .

Figure $3 \mathrm{c}$ displays the current density versus time curves obtained at $1.8 \mathrm{~V}$ vs RHE for the $\mathrm{NiOOH}$ electrocatalyst. The results are similar to those obtained for polycrystalline Pt (see Figure 2c): an increase in the current density is observed when the electrode is rotated and when oxygen is removed from the electrolyte.

It is known that nickel hydroxides can deactivate during long-term measurements. In order to ensure optimal activity of the materials, for each experiment, we performed a new $\mathrm{Ni}(\mathrm{OH})_{2}$ film deposition. The electrodes were cycled until the cyclic voltammogram was stable. The results shown in Figure S3 in the Supporting Information confirm that, without rotation, and in $\mathrm{O}_{2}$-free electrolyte, the deactivation of $\mathrm{NiOOH}$ is faster. When the electrolyte is saturated with $\mathrm{O}_{2}$, the cyclic voltammograms overlap, suggesting good stability of the electrocatalyst surface; however, the curves obtained at rotations of $<1600 \mathrm{rpm}$ (Figure $\mathrm{S} 3 \mathrm{~b}$ in the Supporting Information) and $\mathrm{O}_{2}$-free electrolyte showed a slower decline, indicating good activity and stability of the electrode under these conditions.

\section{DISCUSSION AND CONCLUSION}

The results presented in the previous section show that the presence of oxygen in the electrolyte has no (or a negative) effect on the activity of the oxygen evolution reaction. This exact origin of this negative effect is not entirely clear and may be due to changes in the state of the surface or a consequence of the formation of small bubbles accumulating on the 
electrode surface. ${ }^{25-28}$ In principle, the presence of oxygen in the electrolyte could lead to enhanced bubble formation, since the critical concentration to reach supersaturation is reached earlier, although, practically, the critical concentration may be very much higher than the saturation concentration, such that the effect may be small. ${ }^{29}$ Bubbles can be removed by rotating the electrode or by stirring of the electrolyte when the electrode cannot be rotated. Comparing our results for Pt and $\mathrm{NiOOH}$ electrodes, the effect seems to be more severe for the $\mathrm{NiOOH}$ electrode, which we tentatively ascribe to the more porous structure of the $\mathrm{NiOOH}$ film. ${ }^{28}$ Therefore, saturating the electrolyte with $\mathrm{O}_{2}$ may resemble the conditions in a real electrolyzer more closely.

In addition, an important secondary issue that must be considered during OER measurements is related to the use of the reference electrode. In the presence of oxygen, one must take care to avoid having a mixed reference potential, and confirm the oxygen insensitivity of the reference electrode used.

\section{EXPERIMENTAL SECTION}

Experimental Conditions, Electrodes, and Reactants. Electrochemical measurements were performed at room temperature $\left(20 \pm 1{ }^{\circ} \mathrm{C}\right)$ with an Autolab PGSTAT12 system, using a single-compartment three-electrode cell thatw as composed of fluorinated ethylene propylene (FEP). The cell and all glassware used were rigorously cleaned before starting the experiments by storing overnight in concentrated $\mathrm{H}_{2} \mathrm{SO}_{4}$ to remove metals and organic contaminants. Before use, it was rinsed and boiled five times in water to ensure the cleanness of the system. All water used for cleaning and preparing electrolyte solutions was demineralized and ultrafiltered by a Millipore Milli-Q system (resistivity of $>18.2 \mathrm{M} \Omega$, TOC $<5$ $\mathrm{ppb})$.

The chemicals used in this work were of ultrahigh purity: $\mathrm{Ni}\left(\mathrm{NO}_{3}\right)_{2} \cdot 6 \mathrm{H}_{2} \mathrm{O}$ (Aldrich trace metal basis, 99.999\%), $\mathrm{H}_{2} \mathrm{SO}_{4}$ (Aldrich, 98\%), and $\mathrm{NaOH}$ (Aldrich, $30 \%$ solution in $\mathrm{H}_{2} \mathrm{O}$, TraceSelect for trace analysis).

For the OER measurements, high-area Pt and Au counter electrodes were used (the latter for measurements using $\mathrm{NiOOH}$ as a catalyst). A commercial and self-contained RHE (Gaskatel, Hydroflex), consisting of polytetrafluoroethylene (PTFE), a silver-silver chloride ( $\mathrm{Ag} / \mathrm{AgCl})$, and a mercurymercury oxide $(\mathrm{Hg} / \mathrm{HgO})$, was used as a reference electrode.

The working electrodes were a polycrystalline Pt rotating disk electrode, or a $\mathrm{Ni}$ film deposited on $\mathrm{Au}$ rotating-disk electrode (RDE) $\left(\phi=0.196 \mathrm{~cm}^{2}\right)$. Before measurements, the $\mathrm{RDE}$ electrode was first polished with 0.3 and $0.05 \mu \mathrm{m}$ alumina paste (Buehler, Ltd.). Subsequently, the electrode was sonicated for $5 \mathrm{~min}$ to remove polishing particles. All experiments were performed at 0 or $1600 \mathrm{rpm}$ (unless otherwise stated).

Nickel was plated on the polycrystalline gold electrode by galvanostatic electrodeposition from $5 \mathrm{mM} \mathrm{Ni}\left(\mathrm{NO}_{3}\right)_{2} \cdot 6 \mathrm{H}_{2} \mathrm{O}$ solution, using $0.1 \mathrm{M} \mathrm{NaClO}_{4}$ as a supporting electrolyte. The deposition was carried out by applying a cathodic current (50 $\mu \mathrm{A}$ ) for a given time, in order to obtain ca. five monolayers of coverage; the time for nickel plating was calculated according to the real surface area of the working electrode in order to deposit $5 \times 726 \mu \mathrm{C} \mathrm{cm}^{-2}$, with the latter value corresponding to the charge needed to deposit one monolayer of closely packed metallic nickel from a $\mathrm{Ni}^{\mathrm{II}}$ solution, assuming the atomic radius of $\mathrm{Ni}$ to be $0.124 \mathrm{~nm}$ and a density of $8.908 \mathrm{~g}$ $\mathrm{cm}^{-3} \cdot 30$

All potentials in this work are reported versus the RHE. The potentials were converted to the RHE scale, according to eq 1 :

$$
E_{\mathrm{RHE}}=E+E_{\mathrm{ref}}+0.059 \Delta \mathrm{pH}
$$

where $E_{\mathrm{RHE}}$ is the potential on the RHE scale and $E$ is the experimental potential measured with respect to the reference electrode potential $\left(E_{\text {ref }}\right)$.

For measurements in rigorously $\mathrm{Fe}$-free $\mathrm{NaOH}$ electrolytes, we used a method reported by Boettcher et al., ${ }^{13}$ using a highpurity $\mathrm{Ni}(\mathrm{OH})_{2}$ precipitate as an $\mathrm{Fe}$ absorbent to remove trace Fe from the $\mathrm{NaOH}$ electrolyte. Briefly, it consists of dissolving 99.999\% pure $\mathrm{Ni}\left(\mathrm{NO}_{3}\right)_{2} \cdot 6 \mathrm{H}_{2} \mathrm{O}$ in ultrapure water in a polypropylene tube. Next, $1.0 \mathrm{M} \mathrm{NaOH}$ was added to precipitate high-purity $\mathrm{Ni}(\mathrm{OH})_{2}$. The mixture was shaken and centrifuged, and the supernatant was decanted. The $\mathrm{Ni}(\mathrm{OH})_{2}$ was washed three times by adding water and $1.0 \mathrm{M}$ $\mathrm{NaOH}$, redispersing the solid, centrifuging and decanting the supernatant. Finally, the tube was filled with $1.0 \mathrm{M} \mathrm{NaOH}$ for purification. The solid was redispersed and mechanically agitated for at least $10 \mathrm{~min}$, followed by at least $3 \mathrm{~h}$ of resting. The mixture was centrifuged, and the purified $\mathrm{NaOH}$ supernatant was decanted into a polypropylene bottle for storage, and later used for the electrochemical experiments.

Prior to the electrochemical measurements, the working solution was deaerated with argon for $30 \mathrm{~min}$ and the cyclic voltammograms were collected at different scan rates (1.0, 10.0 , and $50.0 \mathrm{mV} \mathrm{s}^{-1}$ ) with and without rotation. Afterward, the electrolyte was saturated with $\mathrm{O}_{2}$ during $30 \mathrm{~min}$ and the same measurements were performed.

\section{ASSOCIATED CONTENT}

\section{Supporting Information}

The Supporting Information is available free of charge on the ACS Publications website at DOI: 10.1021/acscatal.8b01447.

Cyclic voltammetry curves of platinum and nickel oxyhydroxide electrodes (PDF)

\section{AUTHOR INFORMATION}

\section{Corresponding Author}

*E-mail: m.koper@chem.leidenuniv.nl.

ORCID

Marc T. M. Koper: 0000-0001-6777-4594

Notes

The authors declare no competing financial interest.

\section{ACKNOWLEDGMENTS}

This work was supported by the European Union, under the aleaf project (No. 732840-A-LEAF).

\section{REFERENCES}

(1) Trasatti, S. Electrocatalysis in the Anodic Evolution of Oxygen and Chlorine. Electrochim. Acta 1984, 29, 1503-1512.

(2) Grimaud, A.; May, K. J.; Carlton, C. E.; Lee, Y.-L.; Risch, M.; Hong, W. T.; Zhou, J.; Shao-Horn, Y. Double Perovskites as a Family of Highly Active Catalysts for Oxygen Evolution in Alkaline Solution. Nat. Commun. 2013, 4, 2439.

(3) Burke, M. S.; Enman, L. J.; Batchellor, A. S.; Zou, S.; Boettcher, S. W. Oxygen Evolution Reaction Electrocatalysis on Transition Metal Oxides and (Oxy)hydroxides: Activity Trends and Design Principles. Chem. Mater. 2015, 27, 7549-7558. 
(4) Diaz-Morales, O.; Ferrus-Suspedra, D.; Koper, M. T. M. The Importance of Nickel Oxyhydroxide Deprotonation on Its Activity towards Electrochemical Water Oxidation. Chem. Sci. 2016, 7, 26392645.

(5) Diaz-Morales, O.; Raaijman, S.; Kortlever, R.; Kooyman, P. J.; Wezendonk, T.; Gascon, J.; Fu, W. T.; Koper, M. T. M. Iridium-Based Double Perovskites for Efficient Water Oxidation in Acid Media. Nat. Commun. 2016, 7, 12363.

(6) Walter, M. G.; Warren, E. L; McKone, J. R.; Boettcher, A. W.; Mi, Q.; Santori, E. A.; Lewis, N. S. Solar Water Splitting Cells. Chem. Rev. 2010, 110, 6446-6473.

(7) McCrory, C. C. L.; Jung, S. H.; Peters, J. C.; Jaramillo, T. F. Benchmarking Heterogeneous Electrocatalysts for the Oxygen Evolution Reaction. J. Am. Chem. Soc. 2013, 135, 16977-16987.

(8) Chen, J. G.; Jones, C. W.; Linic, S.; Stamenkovic, V. R. Best Practices in Pursuit of Topics in Heterogeneous Electrocatalysis. ACS Catal. 2017, 7, 6392-6393.

(9) Suntivich, J.; May, K. J.; Gasteiger, H. A.; Goodenough, J. B.; Shao-Horn, Y. A Perovskite Oxide Optimized for Oxygen Evolution Catalysis from Molecular Orbital Principles. Science 2011, 334, 13831385.

(10) Hong, W.; Stoerzinger, K. A.; Lee, Y.-L.; Giordano, L.; Grimaud, A. J. L.; Johnson, A. M.; Hwang, J.; Crumlin, E.; Yang, W.; Shao-Horn, Y. Charge-Transfer-Energy-Dependent Oxygen Evolution Reaction Mechanisms for Perovskite Oxides. Energy Environ. Sci. 2017, 10, 2190-2200.

(11) Wang, P.; Yan, M.; Meng, J.; Jiang, G.; Qu, L.; Pan, X.; Liu, J. Z.; Mai, L. Oxygen Evolution Reaction Dynamics Monitored by an Individual Nanosheet- Based Electronic Circuit. Nat. Commun. 2017, 8,645 .

(12) Hong, W. T.; Risch, M.; Stoerzinger, K. A.; Grimaud, A.; Suntivich, J.; Shao-Horn, Y. Toward the Rational Design of NonPrecious Transition Metal Oxides for Oxygen Electrocatalysis. Energy Environ. Sci. 2015, 8, 1404-1427.

(13) Trotochaud, L.; Young, S. L.; Ranney, J. K.; Boettcher, S. W. Nickel-Iron Oxyhydroxide Oxygen-Evolution Electrocatalysts: The Role of Intentional and Incidental Iron Incorporation. J. Am. Chem. Soc. 2014, 136, 6744-6753.

(14) Birss, V. I.; Damjanovic, A. Oxygen Evolution at Platinum Electrodes in Alkaline Solutions. I Dependence on Solution $\mathrm{pH}$ and Oxide Film Thickness. J. Electrochem. Soc. 1987, 134, 113-117.

(15) Li, Y.; Li, F.; Meng, X.-Y.; Li, S.; Zeng, J.-H.; Chen, Y. Ultrathin $\mathrm{Co} 3 \mathrm{O} 4$ Nanomeshes for the Oxygen Evolution Reaction. ACS Catal. 2018, 8, 1913-1920.

(16) Kim, B.; Oh, A.; Kabiraz, M. K.; Hong, Y.; Joo, J.; Baik, H.; Choi, S.-I.; Lee, K. NiOOH Exfoliation-Free Nickel Octahedra as Highly Active and Durable Electrocatalysts Toward the Oxygen Evolution Reaction in an Alkaline Electrolyte. ACS Appl. Mater. Interfaces 2018, 10, 10115-10122.

(17) Nurlaela, E.; Shinagawa, T.; Qureshi, M.; Dhawale, D. S.; Takanabe, K. Temperature Dependence of Electrocatalytic and Photocatalytic Oxygen Evolution Reaction Rates Using NiFe Oxide. ACS Catal. 2016, 6, 1713-1722.

(18) Chi, J.; Yu, H.; Qin, B.; Fu, L.; Jia, J.; Yi, B.; Shao, Z. Vertically Aligned $\mathrm{FeOOH} / \mathrm{NiFe}$ Layered Double Hydroxides Electrode for Highly Efficient Oxygen Evolution Reaction. ACS Appl. Mater. Interfaces 2017, 9, 464-471.

(19) Kongkanand, A.; Ziegelbauer, J. M. Surface Platinum Electrooxidation in the Presence of Oxygen. J. Phys. Chem. C 2012, 116 (5), 3684-3693.

(20) Matsumoto, M.; Miyazaki, T.; Imai, H. Oxygen-Enhanced Dissolution of Platinum in Acidic Electrochemical Environments. J. Phys. Chem. C 2011, 115 (22), 11163-11169.

(21) Damjanovic, A. Electron Transfer Through Thin Anodic Oxide Films during the Oxygen Evolution Reactions at Pt Electrodes. J. Electrochem. Soc. 1991, 138, 2549-2555.

(22) Friebel, D.; Louie, M. W.; Bajdich, M.; Sanwald, K. E.; Cai, Y.; Wise, A. M.; Cheng, M. J.; Sokaras, D.; Weng, T. C.; Alonso-Mori, R.; Davis, R. C.; Bargar, J. R.; Nørskov, J. K.; Nilsson, A.; Bell, A. T.
Identification of Highly Active $\mathrm{Fe}$ Sites in $(\mathrm{Ni}, \mathrm{Fe}) \mathrm{OOH}$ for Electrocatalytic Water Splitting. J. Am. Chem. Soc. 2015, 137, 1305-1313.

(23) Burke, M. S.; Zou, S.; Enman, L. J.; Kellon, J. E.; Gabor, C. A.; Pledger, E.; Boettcher, S. W. Revised Oxygen Evolution Reaction Activity Trends for First-Row Transition-Metal (Oxy)hydroxides in Alkaline Media. J. Phys. Chem. Lett. 2015, 6, 3737-3742.

(24) Trzésniewski, B. J.; Diaz-Morales, O.; Vermaas, D. A.; Longo, A.; Bras, W.; Koper, M. T. M.; Smith, W. A. In Situ Observation of Active Oxygen Species in Fe-Containing Ni-Based Oxygen Evolution Catalysts: The Effect of $\mathrm{pH}$ on Electrochemical Activity. J. Am. Chem. Soc. 2015, 137, 15112-15121.

(25) Janssen, L. J. J.; Barendrecht, E. The Effect of Electrolytic Gas Evolution on Mass Transfer at Electrodes. Electrochim. Acta 1979, 24, 693-699.

(26) Vogt, H. On the Gas-Evolution Efficiency of Electrodes. II Numerical Analysis. Electrochim. Acta 2011, 56, 2404-2410.

(27) Sequeira, C. A. C.; Santos, D. M. F.; Šljukić, B.; Amaral, L. Physics of Electrolytic Gas Evolution. Braz. J. Phys. 2013, 43, 199208.

(28) Kadyk, T.; Bruce, D.; Eikerling, M. How to Enhance Gas Removal from Porous Electrodes? Sci. Rep. 2016, 6, 38780.

(29) German, S. R.; Edwards, M. A.; Ren, H.; White, H. S. Critical Nuclei Size, Rate, and Activation Energy of H2 Gas Nucleation. J. Am. Chem. Soc. 2018, 140, 4047-4053.

(30) House, J. E. Inorganic Chemistry, 2nd Edition; Elsevier/ Academic Press: Waltham, MA, 2013. 